\title{
Downregulation of IncRNA AWPPH inhibits colon cancer cell proliferation by downregulating GLUT-1
}

\author{
JIE BAI ${ }^{1}$, JIAN XU $^{2}$, JIAN ZHAO $^{2}$ and RUI ZHANG ${ }^{2}$ \\ Departments of ${ }^{1}$ Breast Surgery and ${ }^{2}$ Colorectal Surgery, Cancer Hospital of China Medical University, \\ Liaoning Cancer Hospital and Institute, Shengyang, Liaoning 110042, P.R. China
}

Received July 24, 2018; Accepted May 9, 2019

DOI: $10.3892 / \mathrm{ol} .2019 .10515$

\begin{abstract}
Long non-coding RNA (lncRNA) associated with poor prognosis of hepatocellular carcinoma (AWPPH) serves pivotal roles in bladder cancer and liver cancer; however, to the best of our knowledge, its functionality in colon cancer has not been characterized. The present study aimed to investigate the involvement of IncRNA AWPPH in colon cancer. Serum levels of lncRNA AWPPH and glucose transporter 1 (GLUT-1) in patients with early stage colon cancer and healthy controls were measured by reverse transcription-quantitative polymerase chain reaction (RT-qPCR) and ELISA. Correlation between IncRNA AWPPH and GLUT-1 expression was analyzed by Pearson's correlation coefficient. $\chi^{2}$ test was performed to investigate the associations between serum levels of lncRNA AWPPH and clinical data of patients with colon cancer. IncRNA AWPPH short hairpin RNA and GLUT-1 expression vectors were transfected into colon cancer cells, and the effects on lncRNA AWPPH, GLUT-1 and cell proliferation were detected by RT-qPCR, western blotting and Cell Counting Kit- 8 assay. It was observed that serum levels of IncRNA AWPPH and GLUT-1 were significantly higher in patients with colon cancer patients compared with healthy controls. Serum levels of AWPPH and GLUT-1 were significantly positively correlated in patients with colon cancer. Serum levels of lncRNA AWPPH were associated with the tumor size. Furthermore, AWPPH-silencing significantly inhibited GLUT-1 expression and inhibited cancer cell proliferation. GLUT-1 overexpression promoted cancer cell proliferation and attenuated the inhibitory effects of AWPPH-silencing on cancer cell proliferation. However, GLUT-1 overexpression failed to significantly affect the expression of AWPPH. Therefore, it can be concluded that
\end{abstract}

Correspondence to: Professor Rui Zhang, Department of Colorectal Surgery, Cancer Hospital of China Medical University, Liaoning Cancer Hospital and Institute, 44 Xiaoheyan Road, Dadong, Shengyang, Liaoning 110042, P.R. China

E-mail: tbdnvi1@163.com

Key words: colon cancer, long non-coding RNA associated with poor prognosis of hepatocellular carcinoma, glucose transporter 1 , proliferation a downregulation of lncRNA AWPPH may inhibit colon cancer cell proliferation by downregulating GLUT-1.

\section{Introduction}

Colon cancer, a type of malignant tumor that develops from the large bowel, is one of the most frequently diagnosed cancer types among males and females, and has unacceptable high mortality rates worldwide (1-3). In 2014 and 2015, colon cancer caused $\sim 700,000$ deaths $(2,3)$. Treatment outcomes of colon cancer at early stages are generally satisfactory, and $>95 \%$ patients can live $>5$ years following active treatment (4). However, the majority of patients with colon cancer are diagnosed with existing distant tumor metastasis (5), which lacks radical therapeutic regimens, leading to poor survival outcomes of those patients. Although several risk factors, including advanced age and male sex have been identified for colon cancer, the pathogenesis of this disease remains unclear (1). Therefore, it is important to identify molecular markers for the diagnosis of colon cancer at early stages.

Abnormally accelerated glucose metabolism distinguishes cancer cells from normal cells (6). Glucose metabolism is considered as a promising target for cancer therapy (7). Glucose transporter 1 (GLUT-1), a glucose transporter with critical functions in the transport of glucose across the plasma membranes of mammalian cells, serves a key role in glucose metabolism (8). Levels of GLUT-1 have been reported to be elevated in the development of different types of cancer, including breast cancer and liver cancer $(9,10)$. Overexpression of GLUT-1 not only promotes cancer cell proliferation but also protects cancer cells from stress $(11,12)$. It is understood that GLUT-1, under certain conditions, interacts with different long non-coding RNAs (lncRNAs) to participate in cancer progression $(13,14)$. IncRNA associated with poor prognosis of hepatocellular carcinoma (AWPPH) is a recently reported lncRNA with oncogenic functions in bladder cancer (15) and hepatocellular carcinoma (16). The present study demonstrated that lncRNA AWPPH could interact with GLUT-1 to participate in the regulation of colon cancer cell proliferation.

\section{Materials and methods}

Patients. A total of 134 patients with colon cancer were treated at Liaoning Cancer Hospital and Institute (Shengyang, China) 
between May 2015 and January 2018. Among these patients, 46 were included in the present study to serve as the pateint group. The inclusion criteria were as follows: i) patients with colon cancer at stage I and II (early stage) diagnosed by a pathological test; ii) patients with serum samples in the specimen library of the hospital; ii) patients who could fully understand the experimental protocol; and iv) patients willing to join the study. The exclusion criteria were as follows: i) patients with another severe disease at the time of admission; ii) patients who were treated within 3 months prior admission; and iii) patients with chronic diseases. The current study also included 42 healthy individuals to serve as the control group. The health controls received physiological examinations at Liaoning Cancer Hospital and Institute between May 2015 and January 2018, and all had serum samples in the specimen library of the hospital. The Ethics Committee of Liaoning Cancer Hospital and Institute approved the current study prior to the enrollment of participants. All participants provided written informed consent. No significnat differences in age and sex were identified between the two groups. The basic information of all participants is presented in Table I.

Reverse transcription-quantitative polymerase chain reaction $(R T-q P C R)$. Trizol ${ }^{\circledR}$ reagent (Invitrogen; Thermo Fisher Scientific, Inc.) was used to extract total RNA from serum and in vitro cultured cells. Total RNA samples were subjected to denatured agrose gel electrophoresis to assess the quality. The RNA concentration was measured using a NanoDrop ${ }^{\mathrm{TM}} 2000$ spectrophotometer (Thermo Fisher Scientific, Inc.). RT was performed to synthesize cDNA using SuperScript III Reverse Transcriptase (Thermo Fisher Scientific, Inc.) according to the following conditions: $5 \mathrm{~min}$ at $25^{\circ} \mathrm{C}, 20 \mathrm{~min}$ at $55^{\circ} \mathrm{C}$ and $20 \mathrm{~min}$ at $75^{\circ} \mathrm{C}$. All qPCR reaction systems were prepared using SYBR ${ }^{\circledR}$ Green Real-Time PCR Master mix (Thermo Fisher Scientific, Inc.). The following primers were used: IncRNA-AWPPH forward, 5'-CTGGATGGTCGCTGCTTT TTA-3' and reverse, 5'-AGGGGGATGAGTCGTGATTT-3'; and $\beta$-actin forward, 5'-ACCTCTATGCCAACACAGT-3' and reverse, 5'-AGTACTTGCGCTCAGGAGG-3'. The following thermocycling conditions were used for qPCR: $1 \mathrm{~min}$ at $95^{\circ} \mathrm{C}$, followed by 40 cycles of $20 \mathrm{sec}$ at $95^{\circ} \mathrm{C}$ and $40 \mathrm{sec}$ at $58^{\circ} \mathrm{C}$. The qPCR products were all subjected to agrose gel electrophoresis and some were sequenced to make sure the correct products were obtained. Data normalizations were performed using the $2^{-\Delta \Delta \mathrm{Cq}}$ method (17).

ELISA. Serum levels of GLUT-1 were measured by ELISA using a GLUT-1 ELISA kit (cat. no. MBS721715; MyBioSource).

Cell lines, cell culture and transfection. The normal human colon cell line FHC (cat. no. CRL-1831) and colorectal adenocarcinoma cell line HT-29 (cat. no. HTB-38 ${ }^{\mathrm{TM}}$ ) were purchased from the American Type Culture Colletcion (ATCC). Cells were cultivated with McCoy's 5a Medium Modified (ATCC) containing $10 \%$ fetal bovine serum (ATCC) and placed at $37^{\circ} \mathrm{C}$ in a humidified incubator containing $5 \% \mathrm{CO}_{2}$. $\mathrm{FHC}$ is a normal colon epithelial cell line collected at 13 weeks gestation. IncRNA-AWPPH short hairpin RNA (shRNA; 5'-GGA ATGCAGCTGAAAGATTCC-3') and scrambled shControl (5'-UUUCCGAACGUGUCACGUdTdT-3') were synthesized
Table I. Information for the patient group and control group.

\begin{tabular}{lcc}
\hline Characteristic & $\begin{array}{c}\text { Patient } \\
\text { group }(\mathrm{n}=46)\end{array}$ & $\begin{array}{c}\text { Control } \\
\text { group }(\mathrm{n}=42)\end{array}$ \\
\hline Sex & & \\
Male, $\mathrm{n}$ & 26 & 24 \\
Female, $\mathrm{n}$ & 20 & 18 \\
Age range, years & $23-70$ & $24-72$ \\
Mean age, years & $48.3 \pm 71.1$ & $47.9 \pm 6.4$ \\
\hline
\end{tabular}

by Shanghai GenePharma Co., Ltd.. Full length GLUT-1 cDNA was amplified by PCR reaction. GLUT-1 cDNA was inserted into a pIRSE2 vector (Clontech Laboratories, Inc.) to generate GLUT-1 expression vector. Vectors $(10 \mathrm{nM})$ and shRNA (50 mM) were transfected into $1 \times 10^{6}$ FHC and HT-29 cells using Lipofectamine ${ }^{\circledR} 2000$ reagent (cat. no. 11668-019; Invitrogen; Thermo Fisher Scientific, Inc,). Untransfected cells were used as control cells. Cells transfected with scrambled shControl or empty vector were used as negative control cells. GLUT-1 overexpression and lncRNA AWPPH-silencing were confirmed $12 \mathrm{~h}$ after transfection. Subsequent experiments were performed when the overexpression rate was $>200 \%$ and the knockdown rate was $<50 \%$ compared with the control cells.

Cell proliferation assay. Following transfection and confirmation, cells were harvested and cell suspensions were prepared with a final cell density of $4 \times 10^{4}$ cells/well. Each well of a 96-well plate was filled with $0.1 \mathrm{ml}$ cell suspension containing $4 \times 10^{3}$ cells. The plate was incubated at $37^{\circ} \mathrm{C}$ in a $5 \% \mathrm{CO}_{2}$ incubator, followed by addition of $10 \mu \mathrm{l}$ Cell Counting Kit- 8 (CCK-8) reagent at 24, 48, 72 and 96 h. Cells were cultured for an additional $4 \mathrm{~h}$, followed by measurement of the optical density values at $450 \mathrm{~nm}$ using a microplate reader.

Western blot. RIPA lysis and extraction buffer (Thermo Fisher Scientific, Inc.) was used to extract total protein from FHC and HT-29 cells, followed by measurement of protein concentrations using a BCA assay (Sigma-Aldrich; Merck $\mathrm{KGaA})$. Proteins $(20 \mu \mathrm{g})$ were separated by $12 \%$ SDS-PAGE. After gel transfer onto polyvinylidene difluoride membranes, the membranes were blocked in 5\% skimmed milk for $1 \mathrm{~h}$ at room temperature. Subsequently, the membranes were incubated with the following primary antibodies at $4^{\circ} \mathrm{C}$ overnight: Rabbit anti-human GLUT-1 (1:2,000; cat. no. ab15309; Abcam) and GAPDH antibody (1:1,000; cat. no. ab9485; Abcam). Membranes were then incubated with goat anti-rabbit IgG-HRP secondary antibody (1:1,000; cat. no. MBS435036; MyBioSource) for $2.5 \mathrm{~h}$ at $25^{\circ} \mathrm{C}$. ECL Western Blotting Substrate (Thermo Fisher Scientific, Inc.) was used to develop signal. Data were normalized using ImageJ v1.48 software (National Institutes of Health).

Statistical analysis. All experiments were performed in triplicate. GraphPad Prism 6 software (GraphPad Software Inc.) was used for data analyses and comparisons. Gene expression and cell proliferation data were compared using 



Figure 1. Serum levels of lncRNA AWPPH and GLUT-1 are significantly higher in patients with colon cancer compared with healthy controls. Serum levels of (A) lncRNA AWPPH and (B) GLUT-1 were measured by reverse transcription-quantitative polymerase chain reaction and ELISA, respectively. "P $<0.05$. lncRNA, long non-coding RNA; AWPPH, associated with poor prognosis of hepatocellular carcinoma; GLUT-1, glucose transporter 1.
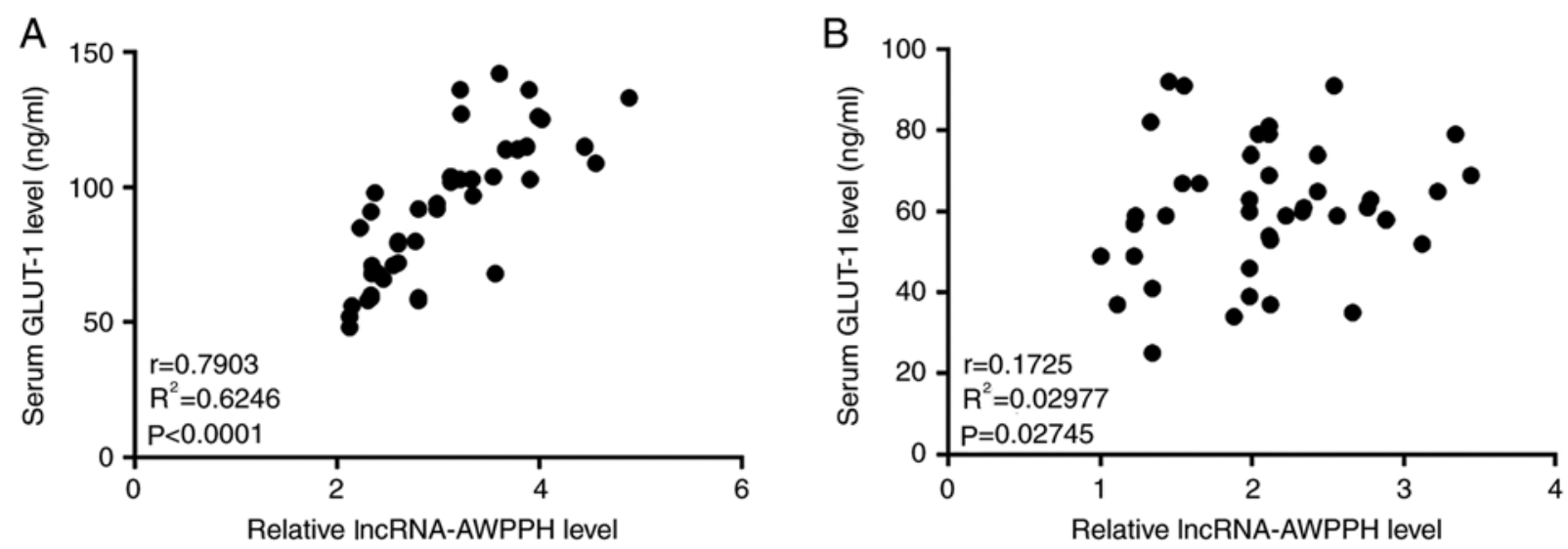

Figure 2. Serum levels of lncRNA AWPPH and GLUT-1 are significantly positively correlated in patients with colon cancer but not in healthy controls. Pearson's correlation coefficient analysis revealed a positive correlation between serum lncRNA AWPPH and GLUT-1 in (A) patients with colon cancer but not in (B) healthy controls. IncRNA, long non-coding RNA; AWPPH, associated with poor prognosis of hepatocellular carcinoma; GLUT-1, glucose transporter 1.

unpaired Student's t-test for two groups and one-way analysis of variance followed by Fisher's least significant difference test for multiple groups. Correlations between serum lncRNA AWPPH and GLUT-1 were analyzed by Pearson's correlation coefficient. $\chi^{2}$ test was used for the analysis between serum levels of lncRNA AWPPH and the clinical data of patients with colon cancer. Data are presented as the mean \pm standard deviation. Receiver operating characteristic curve (ROC) analysis was used to evaluate the diagnostic value of serum lncRNA AWPPH in colon cancer. For this analysis, patients with colon cancer represented the true positive cases and healthy controls represented the true negative cases. $\mathrm{P}<0.05$ was considered to indicate a statistically significant difference.

\section{Results}

Serum levels of IncRNA AWPPH and GLUT-1 are significantly higher in patients with colon cancer compared with healthy controls. RT-qPCR and ELISA demonstrated significantly upregulated serum levels of lncRNA AWPPH $(\mathrm{P}<0.05$; Fig. 1A) and GLUT-1 ( $<<0.05$; Fig. 1B) in patients with colon cancer compared with healthy controls.
Serum levels of AWPPH and GLUT-1 are significantly positively correlated in patients with colon cancer but not in healthy controls. Pearson's correlation coefficient analysis was performed to investigate the potential correlation between serum lncRNA AWPPH and GLUT-1. The results revealed that serum lncRNA AWPPH and GLUT-1 were significantly positively correlated in patients with colon cancer $(\mathrm{P}<0.0001$; Fig. 2A), but not in healthy controls $(\mathrm{P}=0.2745$; Fig. 2B).

Serum levels of AWPPH distinguish patients with colon cancer from healthy controls. ROC analysis was used to evaluate the diagnostic value of serum IncRNA AWPPH for colon cancer. As presented in Fig. 3, the area under the curve (AUC) was 0.8481 , with a $95 \%$ confidence interval of 0.7687-09275 and a standard error of 0.04052 .

Serum levels of IncRNA AWPPH are associated with tumor size. $\chi^{2}$ test was used for the analysis between serum levels of lncRNA AWPPH and clinical data of patients with colon cancer. As presented in Table II, serum levels of lncRNA AWPPH were significantly associated with tumor size $(P=0.04)$, but not age, sex, smoking and drinking habits $(\mathrm{P}>0.05)$. 
Table II. Associations between serum levels of lncRNA AWPPH and clinical data of patients with colon cancer.

\begin{tabular}{|c|c|c|c|c|c|}
\hline Variable & Total, $\mathrm{n}$ & High-expression, $\mathrm{n}$ & Low-expression, $\mathrm{n}$ & $\chi^{2}$ & P-value \\
\hline Sex & & & & 0.35 & 0.55 \\
\hline Male & 26 & 12 & 14 & & \\
\hline Female & 20 & 11 & 9 & & \\
\hline Age, years & & & & 0.79 & 0.37 \\
\hline$\geq 50$ & 21 & 9 & 12 & & \\
\hline$<50$ & 25 & 14 & 11 & & \\
\hline Tumor size, $\mathrm{cm}$ & & & & 4.39 & 0.04 \\
\hline$>5$ & 19 & 13 & 6 & & \\
\hline$\leq 5$ & 27 & 10 & 17 & & \\
\hline Smoking & & & & 0.79 & 0.37 \\
\hline Yes & 21 & 9 & 12 & & \\
\hline No & 25 & 14 & 11 & & \\
\hline Drinking & & & & 0.35 & 0.55 \\
\hline Yes & 22 & 10 & 12 & & \\
\hline No & 24 & 13 & 11 & & \\
\hline
\end{tabular}

lncRNA, long non-coding RNA; AWPPH, associated with poor prognosis of hepatocellular carcinoma.

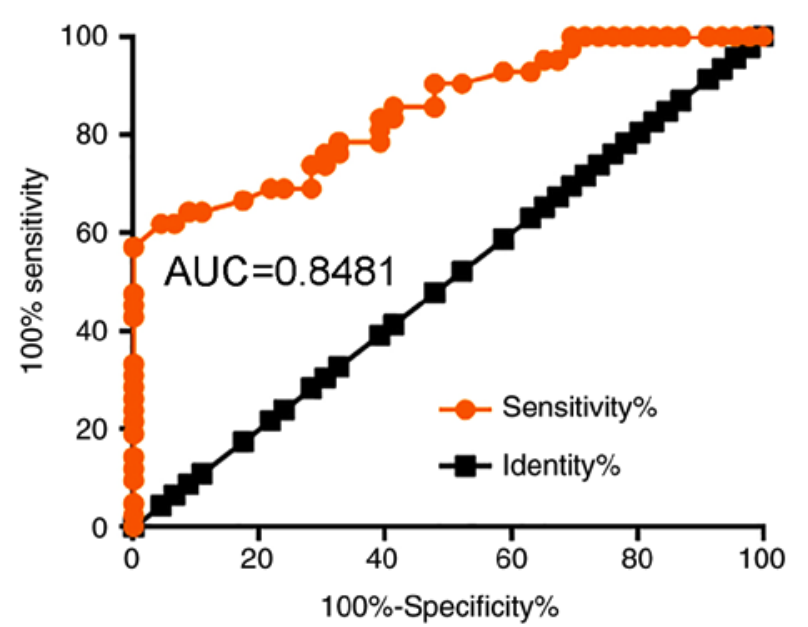

Figure 3. ROC curve analysis of the diagnostic value of serum lncRNA AWPPH for colon cancer. ROC, receiver operating characteristic; lncRNA, long non-coding RNA; AWPPH, associated with poor prognosis of hepatocellular carcinoma.

IncRNA AWPPH-silencing inhibits GLUT-1 expression in colon cancer cells but not in normal colon cells. Cells with lncRNA AWPPH-knockdown were generated and the effects on GLUT-1 expression were investigated. As presented in Fig. 4, compared with the control cells and negative control cells, lncRNA AWPPH-silencing significantly downregulated GLUT-1 in the colorectal adenocarcinoma cell line HT-29 $(\mathrm{P}<0.05)$, but not in the normal FHC colon cell line.

GLUT-1 overexpression attenuated the inhibitory effects of lncRNA AWPPH-silencing on the proliferation of colon cancer cells. Data in Table II revealed the potential involvement of IncRNA AWPPH in the regulation of tumor growth



Figure 4. IncRNA AWPPH-silencing inhibits GLUT-1 expression in colon cancer cells but not in normal colon cells. Western blot analysis was performed to detect GLUT-1 expression in HT-29 and FHC cells following lncRNA AWPPH-silencing. ${ }^{*} \mathrm{P}<0.05$. C, control; NC, negative control; lncRNA, long non-coding RNA; AWPPH, associated with poor prognosis of hepatocellular carcinoma; GLUT-1, glucose transporter 1.

of colon cancer. To further investigate the role of lncRNA AWPPH in the proliferation of colon cancer cells, a CCK-8 assay was performed to detect cell proliferation following lncRNA AWPPH-silencing. AWPPH-silencing and GLUT-1 overexpression were confirmed (Fig. 5). Compared with the control cells, IncRNA AWPPH-silencing significantly inhibited the proliferation of the colorectal adenocarcinoma HT-29 cell line $(\mathrm{P}<0.05)$, but not the normal colon cell line FHC (Fig. 5). In addition, GLUT-1 overexpression promoted cancer cell proliferation and significantly attenuated the inhibitory effects of lncRNA AWPPH-silencing on 
A



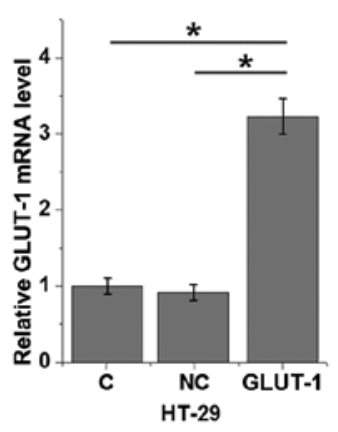



B
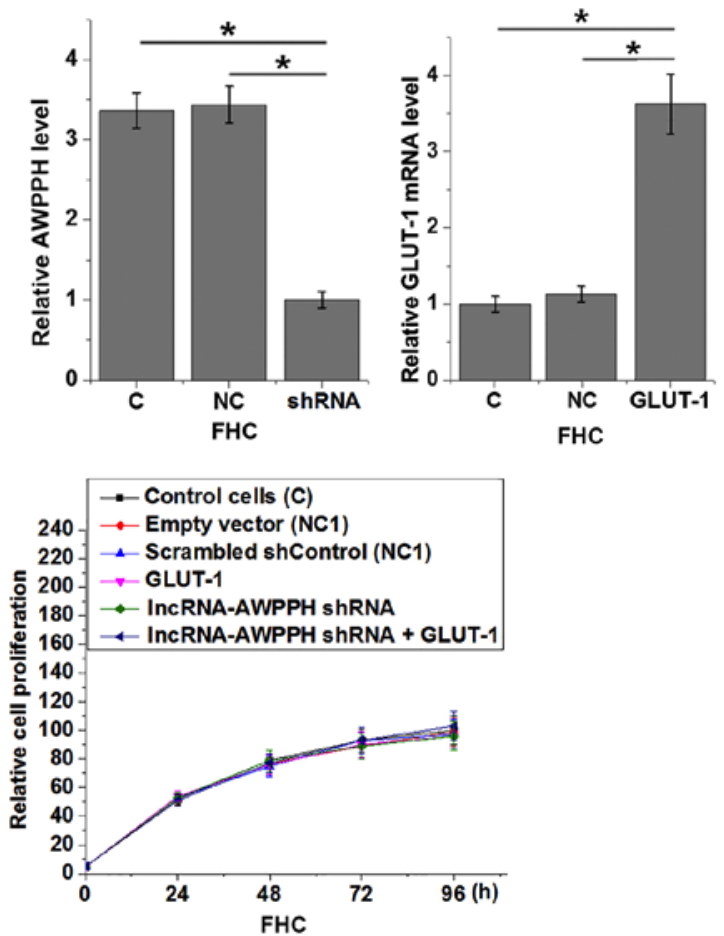

Figure 5. Effects of GLUT-1 overexpression and lncRNA AWPPH-silencing on colon cancer cells and normal colon cells. AWPPH-silencing and GLUT-1 overexpression were confirmed by reverse transcription-quantitative polymerase chain reaction. The effects of AWPPH-silencing and GLUT-1 overexpression on cell proliferation of (A) HT-29 and (B) FHC cells were analyzed by cell proliferation assay. ${ }^{*} \mathrm{P}<0.05$. C, control; NC, negative control; lncRNA, long non-coding RNA; AWPPH, associated with poor prognosis of hepatocellular carcinoma; GLUT-1, glucose transporter 1; shRNA, short hairpin RNA.

the proliferation of the colorectal adenocarcinoma cell line HT-29 $(\mathrm{P}<0.05)$.

\section{Discussion}

The present study used human samples to investigate the involvement of lncRNA AWPPH in colon cancer. The key finding of the current study is that lncRNA AWPPH is involved in the growth of colon cancer. Furthermore, the involvement of lncRNA AWPPH in colon cancer is at least partially mediated by its interactions with GLUT-1.

Treatment of colon cancer is challenged by the fact that the majority of patients are diagnosed with existing distant tumor metastasis (18). Therefore, early diagnosis and treatment is critical. The present study only included patients with stage I or II colon cancer, which are considered early stages of cancer development. It was observed that serum levels of lncRNA AWPPH were significantly higher in patients with colon cancer compared with healthy controls. In addition, a high expression of IncRNA AWPPH could distinguish patients with colon cancer from healthy controls. These data suggest that plasma lncRNA AWPPH may serve as a biomarker for the early diagnosis of colon cancer. However, lncRNA AWPPH is a recently identified lncRNA with an unknown expression pattern in the majority of human diseases. Therefore, multiple diagnostic markers should be combined with lncRNA AWPPH to improve the diagnostic specificity.

Overexpression of GLUT-1 has been observed in different types of human malignancy and inhibition of GLUT-1 is considered to be a promising target for the treatment of human cancer $(19,20)$. Consistent with previous studies, the current study also observed significantly elevated plasma levels of GLUT-1 in patients with colon cancer. Notably, a significant positive correlation was also observed between plasma levels of GLUT-1 and IncRNA AWPPH in patients with colon cancer. In view of the critical functions of GLUT-1 in cancer cell proliferation (21) and the significant association between plasma levels of lncRNA AWPPH and tumor size of colon cancer observed in the present study, it can be hypothesised that IncRNA AWPPH may interact with GLUT-1 to participate in colon cancer cell proliferation. The current in vitro cell experimental data also demonstrated that lncRNA AWPPH and GLUT-1 are involved in the proliferation of colon cancer cells. It can be suggested that lncRNA AWPPH may serve as an upstream activator of GLUT-1 in this process for the following reasons: i) lncRNA AWPPH-silencing downregulated GLUT-1 expression; and ii) GLUT-1 overexpression attenuated the inhibitory effects of IncRNA AWPPH-silencing on colon cancer cell proliferation.

In the present study, lncRNA AWPPH-silencing demonstrated no significant effects on the behaviors of normal colon cells. Notably, GLUT-1 overexpression only promoted the proliferation of colon cancer cells but not the normal colon cells, which may be due to the specific normal colon cancer cell line used in the current study. The present study only revealed a lncRNA AWPPH-GLUT-1 sequential signalling transduction in colon cancer. The interaction between lncRNA-AWPPH and GLUT-1 is likely to be indirect due to the facts that: i) no significant correlation was identified between plasma lncRNA AWPPH and GLUT-1 in healthy 
controls; and ii) lncRNA AWPPH-silencing demonstrated no significant effects on GLUT-1 in normal colon cells.

Two previous studies have reported that lncRNA AWPPH regulates bladder cancer progression by inhibiting SMAD family member 4 via enhancer of zeste homolog 2 (16) and promotes hepatocellular carcinoma progression through Y-box binding protein 1 (18). The present study first reported the involvement of lncRNA AWPPH in cancer biology through the regulation of GLUT-1. This suggests that lncRNA AWPPH may interact with different pathways to participate in different types of cancer.

In conclusion, IncRNA AWPPH and GLUT-1 were demonstrated to be upregulated in patients with colon cancer. In addition, a downregulation of lncRNA AWPPH may inhibit colon cancer cell proliferation by downregulating GLUT-1. However, glucose uptake was not directly measured in the present study. In addition, the sample size was small. Future investigation is needed to solve those problems.

\section{Acknowledgements}

Not applicable.

\section{Funding}

The present study was supported by a National Natural Science Fund from the National Natural Science Foundation of China (grant no. 81672427).

\section{Availability of data and materials}

The analyzed datasets generated during the present study are available from the corresponding author on reasonable request.

\section{Authors' contributions}

JB and RZ designed experiments; JB, JX and JZ performed experiments an collected data. RZ analyzed data and drafted manuscript. All authors approved the manuscript.

\section{Ethics approval and consent to participate}

The present study was approved by the Ethics Committee of Cancer Hospital of China Medical University (Shengyang, China). All patients and healthy volunteers provided written informed consent prior to their inclusion in the study.

\section{Patient consent for publication}

Not applicable.

\section{Competing interests}

The authors declare that they have no competing interests.

\section{References}

1. Marisa L, de Reyniès A, Duval A, Selves J, Gaub MP, Vescovo L, Etienne-Grimaldi MC, Schiappa R, Guenot D, Ayadi M, et al: Gene expression classification of colon cancer into molecular subtypes: Characterization, validation, and prognostic value. PLoS Med 10: e1001453, 2013.
2. Siegel R, Desantis $C$ and Jemal A: Colorectal cancer statistics, 2014. CA Cancer J Clin 64: 104-117, 2014.

3. Torre LA, Bray F, Siegel RL, Ferlay J, Lortet-Tieulent J and Jemal A: Global cancer statistics, 2012. CA Cancer J Clin 65: 87-108, 2015.

4. Lacy AM, García-Valdecasas JC, Delgado S, Castells A, Taurá P, Piqué JM and Visa J: Laparoscopy-assisted colectomy versus open colectomy for treatment of non-metastatic colon cancer: A randomised trial. Lancet 359: 2224-2229, 2002.

5. Benson AB, Bekaii-Saab T, Chan E, Chen YJ, Choti MA, Cooper HS, Engstrom PF, Enzinger PC, Fakih MG, Fenton MJ, et al: Metastatic colon cancer, version 3.2013: Featured updates to the NCCN guidelines. J Natl Compr Canc Netw 11: 141-152, 2013.

6. Annibaldi A and Widmann C: Glucose metabolism in cancer cells. Curr Opin Clin Nutr Metab Care 13: 466-470, 2010.

7. Hamanaka RB and Chandel NS: Targeting glucose metabolism for cancer therapy. J Exp Med 209: 211-215, 2012.

8. Olson AL and Pessin JE: Structure, function, and regulation of the mammalian facilitative glucose transporter gene family. Annu Rev Nutr 16: 235-256, 1996.

9. Pinheiro C, Sousa B, Albergaria A, Paredes J, Dufloth R, Vieira D, Schmitt F and Baltazar F: GLUT1 and CAIX expression profiles in breast cancer correlate with adverse prognostic factors and MCT1 overexpression. Histol Histopathol 26: 1279-1286, 2011

10. Carvalho KC, Cunha IW, Rocha RM, Ayala FR, Cajaíba MM, Begnami MD, Vilela RS, Paiva GR, Andrade RG and Soares FA: GLUT1 expression in malignant tumors and its use as an immunodiagnostic marker. Clinics (Sao Paulo) 66: 965-972, 2011.

11. Oh S, Kim H, Nam K and Shin I: Glut1 promotes cell proliferation, migration and invasion by regulating epidermal growth factor receptor and integrin signaling in triple-negative breast cancer cells. BMB Rep 50: 132-137, 2017.

12. Gonzalez-MenendezP,HeviaD,Alonso-Arias R,Alvarez-ArtimeA, Rodriguez-Garcia A, Kinet S, Gonzalez-Pola I, Taylor N, Mayo JC and Sainz RM: GLUT1 protects prostate cancer cells from glucose deprivation-induced oxidative stress. Redox Biol 17: 112-127, 2018.

13. Wang Y, Zhang X, Wang Z, Hu Q, Wu J, Li Y, Ren X, Wu T, Tao X, Chen X, et al: LncRNA-p23154 promotes the invasion-metastasis potential of oral squamous cell carcinoma by regulating Glut1-mediated glycolysis. Cancer Lett 434: 172-183, 2018.

14. Wei S, Fan Q, Yang L, Zhang X, Ma Y, Zong Z, Hua X, Su D, Sun H, Li H and Liu Z: Promotion of glycolysis by HOTAIR through GLUT1 upregulation via mTOR signaling. Oncol Rep 38: 1902-1908, 2017.

15. Zhu F, Zhang X, Yu Q, Han G, Diao F, Wu C and Zhang Y: LncRNA AWPPH inhibits SMAD4 via EZH2 to regulate bladder cancer progression. J Cell Biochem 119: 4496-4505, 2018.

16. Zhao X, Liu Y and Yu S: Long noncoding RNA AWPPH promotes hepatocellular carcinoma progression through YBX1 and serves as a prognostic biomarker. Biochim Biophys Acta 1863: 1805-1816, 2017.

17. Livak KJ and Schmittgen TD: Analysis of relative gene expression data using real-time quantitative PCR and the 2(-Delta Delta C(T)) method. Methods 25: 402-408, 2001.

18. Riihimäki M, Hemminki A, Sundquist J and Hemminki K: Patterns of metastasis in colon and rectal cancer. Sci Rep 6: 29765, 2016.

19. Kanjanapan Y, Deb S, Young RJ, Bressel M, Mileshkin L, Rischin D, Hofman MS, Narayan K and Siva S: Glut-1 expression in small cervical biopsies is prognostic in cervical cancers treated with chemoradiation. Clin Transl Radiat Oncol 2: 53-58, 2017.

20. Sharen G, Peng Y, Cheng H, Liu Y, Shi Y and Zhao J: Prognostic value of GLUT-1 expression in pancreatic cancer: Results from 538 patients. Oncotarget 8: 19760-19767, 2017.

21. Noguchi C, Kamitori K, Hossain A, Hoshikawa H, Katagi A, Dong Y, Sui L, Tokuda M and Yamaguchi F: D-Allose inhibits cancer cell growth by reducing GLUT1 expression. Tohoku J Exp Med 238: 131-141, 2016.

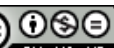

This work is licensed under a Creative Commons Attribution-NonCommercial-NoDerivatives 4.0 International (CC BY-NC-ND 4.0) License. 